\title{
Correspondence
}

\author{
Editors: Andrew C. Smith and Grog Wilkinson
}

\section{The Continuum of Psychosis and the Gene}

SIR: Crow (Journal, October 1986, 149, 419-429) appears to fall into the trap, common to most discussions of unitary psychosis, of regarding psychosis or psychoses as disease(s) rather than syndrome(s). As a result, he is led to postulate the existence of a peculiarly unstable gene or limited set of genes whose abnormalities express themselves as psychosis of varying symptomatology.

That schizophrenia is a syndrome has been recognised ever since Eugen Bleuler wrote about "the schizophrenias", and the evidence that they have multiple aetiologies is now fairly compelling. Of particular relevance to Crow's argument is the finding that process schizophrenias may have a rather low heritability relative to some other types. Severe depression, also, seems to have multiple causes and it is probable that mania has more than one cause (it appears that, in a small number of families, its inheritance is X-linked while in most families it is not; some patients may have abnormal vanadium metabolism; some patients respond well to lithium while others do not, etc.). In situations like this, the observation of continua between syndromes is to be expected and has no great aetiological significance. After all, high dose steroids can produce depression in one patient, mania in another and paranoid schizophrenia in a third. It would be most interesting to know the reason for these idiosyncrasies, but they would hardly justify postulating the existence of a special 'steroid psychosis' gene with unique properties.

It would not be difficult to construct an argument similar to Crow's, centering around bellyache. There is considerable overlap in the symptoms and other characteristics of people suffering abdominal pain, and it may well be the case that children of women with gallstones are prone to develop diverticulitis (as a result of common nutritional habits). Equally, there are probably seasonality effects in bellyache since it is likely to be more common at Christmas time and over the summer holiday period. Perhaps, therefore, there is a bellyache gene similar to the proposed psychosis gene. There are no doubt genes controlling cerebral organisation and dominance effects which may one day be of great interest to psychiatrists especially, I suspect, those studying personality disorder, but existing knowledge is insufficient to enable one to say anything of heuristic value about them.

\section{Royal South Hants Hospital Southampton SO9 4PE}

C. M. H. NunN

SIR: Crow is to be congratulated on his imaginative theory of psychosis (Journal, October 1986, 149, 419-429). One thing puzzles me about his use of the season of birth data to support a viral explanation. His Figure 2 certainly shows, consistent with the hypothesis, that there is an excess of births in patients with psychotic illnesses during the winter months. However, it also shows an equally large reduction during the summer months, a phenomenon that seems to be difficult to reconcile with the presumed pathogenic effects of a virus.

There are several equally convincing explanations of the season of birth effect, one being that it reflects the cycles of sexual activity among the parents of future psychotic patients. Might this not be a more credible and parsimonious explanation of the data, given that there is no other evidence for the viral theory?

\section{Magdalen College}

Gordon Claridge Oxford OX14AU

\section{Chronic Schizophrenia and Long-term Hospitalisation}

SiR: It was good of Abrahamson (Journal, September $1986,149,382)$ to notice Institutionalism and Schizophrenia (Wing \& Brown, 1970), but I wonder if I could persuade him to re-read it? He will find that we did not describe one pattern of relationship between negative aspects of behaviour in schizophrenia and length of stay in hospital. There were three, a different pattern for each of our hospitals. More important, our conclusions about the influence of social environment were not derived from these results. On the contrary, we stated (p. 12) that there was overwhelming evidence against the "startling assumption that length of stay is in any way significant as a measure of severity of illness".

What we did find was that when age and length of stay were standardised, patients in the hospital with 
the poorest social conditions still had the most severe deficit. Moreover, when twenty patients from each hospital, matched for age, length of stay, clinical condition, negative score and attitude to discharge, were compared, major differences remained between the social environments provided for them by the hospitals.

Among much other evidence provided, Abrahamson may be surprised to read that this was not just a cross-sectional study but involved repeated observations over a period of eight years. The social withdrawal scores rated at the beginning and end of that time were highly correlated and showed a mild average improvement in those who remained in hospital. His observation that schizophrenic patients do not necessarily deteriorate thus replicates ours. However, we did measure an increase in 'social poverty' in two of the hospitals during certain periods of observation, with a concomitant increase in "clinical poverty'.

We pointed out that surveys of this kind could not provide definitive tests of the three kinds of theory stated in chapter 1 , but this unit has provided a series of other studies, some of them experimental, that strongly support an interactive socio-medical model. Abrahamson might be particularly interested in an experiment involving very severely impaired patients (Wing \& Freudenberg, 1961). They were not impervious to further social stimulation, the problem was to maintain improvement once it was withdrawn.

\section{MRC Social Psychiatry Unit Institute of Psychiatry \\ De Crespigny Park \\ London SE5 8 AF}

\section{Roferences}

WING, J. K. \& Brown, G. (1970) Institutionalism and Schizophrenia. London: Cambridge University Press.

- \& FreudenberG, R. K. (1961) The response of severely ill chronic schizophrenic patients to social stimulation. American Journal of Psychiatry, 118, 311-321.

\section{Gluten Sensitivity in Schizophrenis}

SIR: Vlissides, Venulet \& Jenner (Journal, A pril 1986 $148,447-452$ ) have concluded that gluten sensitivity is of little importance for schizophrenia in general. This far-reaching conclusion is based on a study which is methodologically unsound and, at best, inconclusive.

The difficulty in interpreting the data on quite remarkable patient improvement noted after the switch from pre-trial hospital diet to gluten-free diet is a case in point. Five parameters showed statisti- cally significant improvement: psychotic disorganisation, retardation, hostile belligerence, anxious depression, and depressive mood. No significant worsening was noted on any parameter. Judging from Figure 1, several of the remaining seven PIP dimensions may well have registered significant improvement under gluten-free diet had one-tailed (directional) statistical tests been performed, which is the appropriate statistical technique since a specific hypothesis was under investigation. However, regardless of the statistical method, one is unable to determine whether the data reflect treatment effect, observer bias or a placebo effect because the period before gluten-free diet was non-blind and there was no parallel control group. The subsequent period of gluten challenge did not do much to resolve the issue because possible observer bias arising from knowledge of the sequence of treatments and the time series effects were not controlled. More importantly, the research design did not permit a consideration of the possibility that the six week gluten challenge, while not worsening psychopathology to base-line values, may well have had enough adverse effect in the time period to arrest therapeutic progress. In the AB research design used, this would show up as 'no change', whereas in the ABA design used in the Singh \& Kay (1976) study, such an adverse effect would very likely have been detected.

The authors attributed the clinical improvement in patients to the attention they received during the study, or what would generally be described as a placebo effect. Within the framework of their study design, such a conclusion would be no more valid than that suggesting an active treatment effect of gluten exclusion. To the contrary, sustained improvement of a highly chronic and treatment-resistant group of schizophrenics lasting over 3 months due to mere attention or placebo effect would be highly implausible indeed.

The study is also open to criticism because of the patient sample used. While the hypothesis being tested relates to schizophrenia, 7 of the 24 patients in the study had other diagnoses. Of the 17 schizophrenics, only one had a diagnosis of non-paranoid schizophrenia, which is the classification suggested by previous work to be at greater risk for gluten sensitivity (Singh, 1979). On top of that, the population was highly chronic and one in which likelihood of response to any form of treatment would be small.

We conclude, therefore, that the study is not a valid test of the gluten hypothesis of schizophrenia and that the conclusions reached by the authors with regard to the hypothesis are not warranted. In an area of such importance as schizophrenia, this 